\title{
Herbal Cures Practised By Rural Populace In Varanasi Region Of Eastern U.P.(India)
}

\author{
Sanjay Srivastava ${ }^{1}$ and C.O.Samuel ${ }^{2}$ \\ ${ }^{I}$ Deptt. Of Botany,Harish Chandra P.G. College,Varanasi (U.P.)India. \\ ${ }^{2}$ Deptt. Of Botany,St.Andrew's College, Gorakhpur (U.P.)India.
}

\begin{abstract}
A survey based study to collect information regarding use of herbs as household treatment of common ailments in rural areas of Varanasi region of eastern U.P. was undertaken .In Varanasi as in other parts of India, the people especially those residing in rural and semi-urban areas still practise herbal cures for many of their ailments. In the present investigation a total of 40 medicinally important plant species belonging to 27 families were recorded which are frequently used by local populace to cure diseases such as cold,cough,fever,snake bite,boils piles etc.As plants are easily available and sometimes the only source of healthcare available to poor therefore there is a great need for preservation of such medicinal plants.
\end{abstract}

Keywords: Ayurvedic, healthcare, herbal cure, medicinal plants.

\section{Introduction}

Medicinal plants and plant derived medicines have long been in-use in traditional cultures all over the world. In modern society too, these herbal remedies are increasingly being commercialized. Natural products and their derivatives (including antibiotics) represent more than 50\% of all drugs in clinical use in the world. Higher plants contribution to this is not less than $25 \%$ [1].

The value and importance of traditional knowledge are now being increasingly acknowledged all over the world.The pharmaceutical industry through its research and development activities continues to investigate and confirm the efficacy of many medicines and toxins used by traditional communities [2 and 3].

Indians have one of the world's richest medicinal plants' heritage. Around 8,000 species of plants referred to by over 2,00,000 vernacular names, are used by the people of our country in local healthcare cultures for human, veterinary and agriculture ( bio-fertilizers, seed treatments and bio-pesticides) related applications in country's 10 bio-geographical zones , 25 biotic provinces and 4635 ethnic communities.

Ayurveda ( Indian system of medication) is derived from the Indian words ayur (life) and veda ( knowledge or science) and thus means "the science of life". It is one of oldest system of medication prevailing throughout the globe. The principles of ayurvedic medicines and the medicinal uses of herbs are contained in thousands of poetic hymns in the Rig veda. Knowledge about herbs is also documented in the Atharva veda and in the later works such as the Charak Samhita and the Sushruta Samhita.

The knowledge and wisdom regarding these herbal remedies were passed from one generation to the next through songs and poems, which vidyarthese ( scholars) and local Vaidyas (physicians ) used to learn by heart and recite regularly. The succeeding generations used to modify and enrich this knowledge by their own experiences. Gradually, herbal cure got woven in the very socio-cultural fabric of the society as a whole . As majority of the populace have bare means of livelihood, herbal cure provides safe and effective alternative for treatment of common ailments at almost no cost.

Varanasi is one of the oldest continuously inhabited cities of the world and one of the most important Hindu pilgrimage centres. Incidently, this region of eastern U.P. also has a rich biodiversity of plants. The district lies at $82^{\circ} 50^{\prime} \mathrm{E}$ to $83^{\circ} 03^{\prime}$ and longitude $25^{\circ} 10^{\prime} \mathrm{N}$ to $25^{\circ} 25^{\prime} \mathrm{N}$ at an altitude of approximately $79.1 \mathrm{~m}$ above the sea level, with fairly level topography. The climate of the region is humid subtropical type with high variation between summer and winter temperatures. Summers are long, from early April till October, with intervening monsoon season. Cold waves from the Himalayas lower the region's temperature in the winter from December to February. The average temperature is $32^{\circ}-46^{\circ} \mathrm{C}$ in summer and $5^{\circ}-15^{\circ} \mathrm{C}$ in the winter. The average annual rainfall is $1110 \mathrm{~mm}$. Foggy weather is quite common in winter while hot dry winds called loo blow across the region in summers. The soils of the region are generally old alluvial deposits of the middle gangetic plains. As in other parts of India, Varanasi region also has a long history of use of plants for healthcare.

\section{Materials And Methods}

For the purpose of the present study, several areas of the Varanasi region were surveyed. The areas included several blocks of Varanasi district. Several villages occurring in Phulpur , Sindhora, Cholapur, Babatpur, Baragaon, Kapsethi, Shivpur, Sarnath , Ramnagar and Mirzamurad blocks were surveyed during the course of the study. 
Field trips were organized in different rural and semi-urban areas at regular intervals in different seasons . Different floras were used for the identification of plants [4and 5]. The data collected in the field were formatted and preserved. The medicinal plants were enumerated alphabetically, with their botanical names , families, vernacular names followed by parts used, ailment and mode of administration.

Information regarding medicinal uses of plants were collected from the literature available in the college library and also through internet. The data so collected was cross examined through interviews with local inhabitants of the villages. In many villages local practitioners of traditional medicines called vaidya or kaviraj were convinced to share their knowledge regarding jadi buti (dried and preserved plant parts as called in local language). The interviews conducted were fairly random. All the participants shared their information willingly although their prior consent regarding the interview were also taken.

\section{Results (Table)}

The data collected through surveys and discussion have been tabulated as follows :

\begin{tabular}{|c|c|c|c|c|c|c|}
\hline $\begin{array}{l}\mathbf{S} . \\
\mathbf{N} \\
\mathbf{0 .}\end{array}$ & $\begin{array}{l}\text { Botanical } \\
\text { name }\end{array}$ & Family & $\begin{array}{l}\text { Vernacul } \\
\text { ar name }\end{array}$ & $\begin{array}{l}\text { Parts } \\
\text { used }\end{array}$ & Ailments & $\begin{array}{l}\text { Mode of } \\
\text { administratio } \\
\text { n }\end{array}$ \\
\hline 1. & $\begin{array}{l}\text { Achyranthes } \\
\text { aspera Linn. }\end{array}$ & $\begin{array}{l}\text { Amarantacea } \\
\mathrm{e}\end{array}$ & Chichidi & $\begin{array}{l}\text { Roots } \\
\text { and } \\
\text { leaves }\end{array}$ & Snake bite & $\begin{array}{l}\text { Paste of parts } \\
\text { is applied as } \\
\text { antidote. Half } \\
\text { teaspoon } \\
\text { powdered leaf } \\
\text { is given in } \\
\text { stomach pain. }\end{array}$ \\
\hline 2. & $\begin{array}{l}\text { Adhatoda } \\
\text { vasica Nees. }\end{array}$ & Acanthaceae & Vasak & leaves & Arthiritis & $\begin{array}{l}2 \text { teaspoon } \\
\text { leaf extract } \\
\text { given twice } \\
\text { daily. }\end{array}$ \\
\hline 3. & $\begin{array}{l}\text { Allium } \\
\text { sativum Linn. }\end{array}$ & Liliaceae & Lahsun & Bulb & Arthiritis & $\begin{array}{l}1 \text { teaspoon } \\
\text { bulb juice } \\
\text { given once } \\
\text { daily. }\end{array}$ \\
\hline 4. & $\begin{array}{l}\text { Aloe } \\
\text { barbadensis } \\
\text { Mill. }\end{array}$ & Liliaceae & $\begin{array}{l}\text { Gheekwar } \\
\text { /gwarpath } \\
\text { a }\end{array}$ & Leaves & $\begin{array}{l}\text { Piles, rectal } \\
\text { fissures,rheumatism,conspitatio } \\
\text { n, fever,anthelminthic,menstrual } \\
\text { disorders, hepatoprotective }\end{array}$ & $\begin{array}{l}\text { Pulp of leaves } \\
\text { given orally. }\end{array}$ \\
\hline 5. & $\begin{array}{l}\text { Amarantus } \\
\text { spinosus L. }\end{array}$ & $\begin{array}{l}\text { Amarantacea } \\
\mathrm{e}\end{array}$ & $\begin{array}{l}\text { Katili } \\
\text { chauli }\end{array}$ & $\begin{array}{l}\text { Roots } \\
\text { and } \\
\text { leaves }\end{array}$ & $\begin{array}{l}\text { Antibillious, antifatigus, } \\
\text { inflammation, hemorrhagic } \\
\text { disease, diarrhea , lucorrhoea, } \\
\text { gonorrhea }\end{array}$ & $\begin{array}{l}\text { Oral } \\
\text { administration } \\
\text { of leaf and } \\
\text { root extract }\end{array}$ \\
\hline 6. & $\begin{array}{l}\text { Asparagus } \\
\text { racemosus } \\
\text { (Willd.) } \\
\end{array}$ & Liliaceae & Satavar & Roots & Gout & $\begin{array}{l}\text { Root extract } \\
\text { taken with } \\
\text { water }\end{array}$ \\
\hline 7. & $\begin{array}{l}\text { Boerhaavia } \\
\text { diffusa Linn. }\end{array}$ & $\begin{array}{l}\text { Nyctaginacea } \\
\mathrm{e}\end{array}$ & Purnava & $\begin{array}{l}\text { Whole } \\
\text { plant }\end{array}$ & Elephantiasis & $\begin{array}{l}\text { Plant extract } \\
\text { is applied to } \\
\text { effected part } \\
\text { of leg }\end{array}$ \\
\hline 8. & $\begin{array}{l}\text { Cassia fistula } \\
\text { Linn. }\end{array}$ & $\begin{array}{l}\text { Caesalpiniace } \\
\text { ae }\end{array}$ & Amaltas & $\begin{array}{l}\text { Seeds, } \\
\text { stem } \\
\text { and } \\
\text { bark }\end{array}$ & Dysentery & $\begin{array}{l}\text { Half tea spoon } \\
\text { of seed juice } \\
\text { is } \\
\text { administered } \\
\text { for two days }\end{array}$ \\
\hline 9. & $\begin{array}{l}\text { Cissus } \\
\text { quandrangula } \\
\text { ris L. }\end{array}$ & Vitaceae & Hadjod & $\begin{array}{l}\text { Whole } \\
\text { plant }\end{array}$ & Bone fractures & $\begin{array}{l}\text { Plant paste is } \\
\text { applied on the } \\
\text { fracture part }\end{array}$ \\
\hline 10 & $\begin{array}{l}\text { Curcuma } \\
\text { domestica } \\
\text { Linn. }\end{array}$ & $\begin{array}{l}\text { Zingiberacea } \\
\mathrm{e}\end{array}$ & Haldi & $\begin{array}{l}\text { Rhizo } \\
\text { me }\end{array}$ & Arthritis & $\begin{array}{l}\text { Powdered } \\
\text { rhizome is } \\
\text { taken with } \\
\text { cow's milk }\end{array}$ \\
\hline 11 & $\begin{array}{l}\text { Chenopodium } \\
\text { ambrosioides } \\
\text { Linn. }\end{array}$ & $\begin{array}{l}\text { Chenopodiac } \\
\text { eae }\end{array}$ & $\begin{array}{l}\text { Bara } \\
\text { Bathua }\end{array}$ & $\begin{array}{l}\text { Leaves } \\
\text {, stem }\end{array}$ & Scabies & $\begin{array}{l}\text { Leaves } \\
\text { together with } \\
\text { stem are } \\
\text { boiled in } \\
\text { water }\end{array}$ \\
\hline
\end{tabular}


Herbal Cures Practised By Rural Populace In Varanasi Region Of Eastern U.P.(India)

\begin{tabular}{|c|c|c|c|c|c|c|}
\hline 12 & $\begin{array}{l}\text { Catharanthus } \\
\text { roseus L. }\end{array}$ & Apocynaceae & Sadabahar & Leaves & Diabetes & $\begin{array}{l}\text { Leaves dried } \\
\text { and powdered } \\
\text { and taken } \\
\text { with water }\end{array}$ \\
\hline 13 & $\begin{array}{l}\text { Cleome } \\
\text { viscosa } \mathrm{L} .\end{array}$ & $\begin{array}{l}\text { Capparidacea } \\
\text { e }\end{array}$ & Hurhur & Seeds & Body pain & $\begin{array}{l}\text { Dried seed } \\
\text { powdered } \\
\text { taken with } \\
\text { water }\end{array}$ \\
\hline 14 & $\begin{array}{l}\text { Crinum } \\
\text { asiaticum L. }\end{array}$ & $\begin{array}{l}\text { Amaryllidace } \\
\text { ae }\end{array}$ & Sudarshan & Bulbs & Rheumatism and piles & $\begin{array}{l}\text { Crushed and } \\
\text { roasted bulbs } \\
\text { are eaten }\end{array}$ \\
\hline 15 & $\begin{array}{l}\text { Calotropis } \\
\text { procera } \\
\text { Aiton. }\end{array}$ & $\begin{array}{l}\text { Asclepiadace } \\
\text { ae }\end{array}$ & Madar & Latex & Bodyache & $\begin{array}{l}\text { Latex mixed } \\
\text { in mustard oil } \\
\text { is applied } \\
\text { externally on } \\
\text { the affected } \\
\text { part }\end{array}$ \\
\hline 16 & $\begin{array}{l}\text { Coccinea } \\
\text { indica Wight } \\
\text { \& Arn. }\end{array}$ & $\begin{array}{l}\text { Cucurbitacea } \\
\text { e }\end{array}$ & Kundru & Leaves & Diabetes, fever and asthma & $\begin{array}{l}\text { Leaf extract } \\
\text { mixed in } \\
\text { honey and } \\
\text { given orally. }\end{array}$ \\
\hline $\begin{array}{l}17 \\
.\end{array}$ & $\begin{array}{l}\text { Cyperus } \\
\text { rotundus } \mathrm{L} .\end{array}$ & Cyperaceae & Motha & $\begin{array}{l}\text { Whole } \\
\text { plant }\end{array}$ & Malarial fever & $\begin{array}{l}2 \text { teaspoon } \\
\text { full decoction } \\
\text { of whole plant } \\
\text { given twice } \\
\text { daily. }\end{array}$ \\
\hline 18 & $\begin{array}{l}\text { Datura } \\
\text { innoxia Mill. }\end{array}$ & Solanaceae & Dhatura & Leaves & Respiratory problems & $\begin{array}{l}\text { Dried leaves } \\
\text { smoked in } \\
\text { respirator } \\
\text { problems. }\end{array}$ \\
\hline 19 & $\begin{array}{l}\text { Desmodium } \\
\text { gangeticum } \\
\text { (L.) DC. }\end{array}$ & Fabaceae & Shalparni & Leaves & Hair problems & $\begin{array}{l}\text { Paste of } \\
\text { leaves applied } \\
\text { on scalp to } \\
\text { prevent hair } \\
\text { loss. }\end{array}$ \\
\hline 20 & $\begin{array}{l}\text { Euphorbia } \\
\text { hirta L. }\end{array}$ & $\begin{array}{l}\text { Euphorbiacea } \\
\text { e }\end{array}$ & Duddhi & $\begin{array}{l}\text { Whole } \\
\text { plant }\end{array}$ & Gonorrhoea & $\begin{array}{l}\text { 3-4 teaspoon } \\
\text { of plant juice } \\
\text { and one } \\
\text { teaspoon } \\
\text { sugar in one } \\
\text { cup of warm } \\
\text { milk taken } \\
\text { once a week. }\end{array}$ \\
\hline 21 & $\begin{array}{l}\text { Eclipta alba } \\
\text { (L.) Hassk. }\end{array}$ & Asteraceae & $\begin{array}{l}\text { Bhangraiy } \\
\text { a }\end{array}$ & Leaves & Dandruff treatment & $\begin{array}{l}\text { Leaves boiled } \\
\text { with seeds of } \\
\text { Foeniculum } \\
\text { vulgare in } \\
\text { coconut oil- } \\
\text { the oil extract } \\
\text { applied on } \\
\text { scalp daily. }\end{array}$ \\
\hline 22 & $\begin{array}{l}\text { Helianthus } \\
\text { annuus L. }\end{array}$ & Asteraceae & $\begin{array}{l}\text { Surajmuk } \\
\text { hi }\end{array}$ & $\begin{array}{l}\text { Leaves } \\
\text { and } \\
\text { seeds }\end{array}$ & Cough and fever & $\begin{array}{l}\text { Powdered } \\
\text { seeds taken } \\
\text { with honey to } \\
\text { clear cough } \\
\text { and leaf } \\
\text { extract given } \\
\text { in fever. }\end{array}$ \\
\hline 23 & $\begin{array}{l}\text { Ipomoea } \\
\text { carnea Jace. }\end{array}$ & $\begin{array}{l}\text { Convolvulace } \\
\text { ae }\end{array}$ & Vilaiti ak & $\begin{array}{l}\text { Whole } \\
\text { plant }\end{array}$ & Inducing conception & $\begin{array}{l}\text { Powdered } \\
\text { plant parts } \\
\text { taken along } \\
\text { with pulp of } \\
\text { leaves of Aloe } \\
\text { vera. }\end{array}$ \\
\hline 24 & Jatropha & Euphorbiacea & Ratanjot & Seed & Rheumatic pain & Seed oil is \\
\hline
\end{tabular}


Herbal Cures Practised By Rural Populace In Varanasi Region Of Eastern U.P.(India)

\begin{tabular}{|c|c|c|c|c|c|c|}
\hline . & curcas Linn. & $\mathrm{e}$ & & oil & & $\begin{array}{l}\text { gently } \\
\text { applied on } \\
\text { affected parts. }\end{array}$ \\
\hline 25 & $\begin{array}{l}\text { Murraya } \\
\text { koenigii L. }\end{array}$ & Rutaceae & $\begin{array}{l}\text { Meethi } \\
\text { neem }\end{array}$ & $\begin{array}{l}\text { Leaves } \\
\text { and } \\
\text { roots }\end{array}$ & $\begin{array}{l}\text { Skin eruptions and blood } \\
\text { disorders }\end{array}$ & $\begin{array}{l}\text { Paste of } \\
\text { leaves applied } \\
\text { on skin } \\
\text { eruptions and } \\
\text { bruised skin ; } \\
\text { root extract } \\
\text { given in blood } \\
\text { disorders. }\end{array}$ \\
\hline 26 & $\begin{array}{l}\text { Moringa } \\
\text { pterygosperm } \\
a \text { Gaertn. }\end{array}$ & Moringaceae & Munga & Leaves & Cold and cough & $\begin{array}{l}\text { Leaf extract } \\
\text { given with } \\
\text { honey. }\end{array}$ \\
\hline 27 & $\begin{array}{l}\text { Mentha } \\
\text { piperata } \mathrm{L} .\end{array}$ & Lamiaceae & $\begin{array}{l}\text { Peppermi } \\
\text { nt }\end{array}$ & Leaves & Stomach disorders & $\begin{array}{l}\text { Leaf extract } \\
\text { given with } \\
\text { water or } \\
\text { honey. }\end{array}$ \\
\hline 28 & $\begin{array}{l}\text { Mimosa } \\
\text { pudica } \text { Linn. }\end{array}$ & Mimosaceae & Lajwanti & Leaves & Wound healing and eczema & $\begin{array}{l}\text { Leaf paste } \\
\text { applied on the } \\
\text { affected parts. }\end{array}$ \\
\hline 29 & $\begin{array}{l}\text { Ocimum } \\
\text { sanctum Linn. }\end{array}$ & Lamiaceae & Tulsi & Leaves & $\begin{array}{l}\text { Bronchitis, asthma and malarial } \\
\text { fever }\end{array}$ & $\begin{array}{l}\text { Decoction of } \\
\text { leaves given } \\
\text { twice daily. }\end{array}$ \\
\hline 30 & $\begin{array}{l}\text { Oxalis } \\
\text { corniculata } \mathrm{L} .\end{array}$ & Oxalidaceae & Teenpatia & Leaves & Bloody diarrhoea & $\begin{array}{l}2-3 \text { teaspoon } \\
\text { full of leaf } \\
\text { juice given } \\
\text { thrice daily. }\end{array}$ \\
\hline 31 & $\begin{array}{l}\text { Psoralea } \\
\text { corylifolia } \mathrm{L} .\end{array}$ & Fabaceae & Bakuchi & Seeds & Abcess, boils, carbuncle & $\begin{array}{l}\text { Seed paste } \\
\text { applied on the } \\
\text { affected parts. }\end{array}$ \\
\hline 32 & $\begin{array}{l}\text { Phyllanthus } \\
\text { niruri L. }\end{array}$ & $\begin{array}{l}\text { Euphorbiacea } \\
\text { e }\end{array}$ & $\begin{array}{l}\text { Bhumi } \\
\text { amla }\end{array}$ & $\begin{array}{l}\text { Whole } \\
\text { plant / } \\
\text { Leaves }\end{array}$ & $\begin{array}{l}\text { Liver disorders } \\
\text { ( hepatitis , cirrhosis ) }\end{array}$ & $\begin{array}{l}\text { Leaf or whole } \\
\text { plant extract } \\
\text { is given. }\end{array}$ \\
\hline 33 & $\begin{array}{l}\text { Rauvolfia } \\
\text { serpentina } \\
\text { (L.)Benth. } \\
\text { ex Kurz }\end{array}$ & Apocynaceae & $\begin{array}{l}\text { Sarpagand } \\
\text { ha }\end{array}$ & Roots & $\begin{array}{l}\text { High blood pressure, antidote to } \\
\text { snakebite }\end{array}$ & $\begin{array}{l}\text { Powdered root } \\
\text { is taken with } \\
\text { water for } \\
\text { three days. }\end{array}$ \\
\hline 34 & $\begin{array}{l}\text { Sida cordifolia } \\
\text { L. }\end{array}$ & Malvaceae & Bariyari & $\begin{array}{l}\text { Leaves } \\
\text { and } \\
\text { roots }\end{array}$ & Boils & $\begin{array}{l}\text { Paste of } \\
\text { leaves or roots } \\
\text { applied } \\
\text { externally on } \\
\text { boils for three } \\
\text { days-twice a } \\
\text { day. }\end{array}$ \\
\hline 35 & $\begin{array}{l}\text { Saraca indica } \\
\text { L. }\end{array}$ & $\begin{array}{l}\text { Caesalpiniace } \\
\text { ae } \\
\text { (Leguminosa } \\
\text { e ) }\end{array}$ & Seita asok & $\begin{array}{l}\text { Stem } \\
\text { bark }\end{array}$ & $\begin{array}{l}\text { Bacterial, fungal infections of } \\
\text { skin and tumorous outgrowth }\end{array}$ & $\begin{array}{l}\text { Bark paste } \\
\text { applied on } \\
\text { affected part. }\end{array}$ \\
\hline 36 & $\begin{array}{l}\text { Solanum } \\
\text { nigrum } \mathrm{L} .\end{array}$ & Solanaceae & Makoi & $\begin{array}{l}\text { Fruits } \\
\text { and } \\
\text { roots }\end{array}$ & Liver diseases & $\begin{array}{l}\text { Fruits and } \\
\text { roots are } \\
\text { crushed } \\
\text { together and } \\
\text { the juice is } \\
\text { taken orally } \\
\text { for three days. }\end{array}$ \\
\hline 37 & $\begin{array}{l}\text { Tinospora } \\
\text { cordifolia(Thu } \\
\text { nb.)Miers }\end{array}$ & $\begin{array}{l}\text { Menispermac } \\
\text { eae }\end{array}$ & $\begin{array}{l}\text { Giloy / } \\
\text { guruch }\end{array}$ & $\begin{array}{l}\text { Whole } \\
\text { plant }\end{array}$ & Rheumatic fever, heart problem & $\begin{array}{l}\text { Decoction of } \\
\text { whole plant is } \\
\text { taken orally. }\end{array}$ \\
\hline 38 & $\begin{array}{l}\text { Terminalia } \\
\text { arjuna } \\
\text { (Roxb.) Wight } \\
\text { and Arn. }\end{array}$ & $\begin{array}{l}\text { Combretacea } \\
\text { e }\end{array}$ & Arjun & Bark & Heart, liver diseases & $\begin{array}{l}\text { Powdered } \\
\text { bark is taken } \\
\text { orally with } \\
\text { water or }\end{array}$ \\
\hline
\end{tabular}




\begin{tabular}{|l|l|l|l|l|l|l|}
\hline 39 & $\begin{array}{l}\text { Vernonia } \\
\text { cinerea L. }\end{array}$ & Asteraceae & Sahdevi & $\begin{array}{l}\text { Whole } \\
\text { plant }\end{array}$ & Breast tumor & $\begin{array}{l}\text { honey. } \\
\text { whole plant is } \\
\text { heated along } \\
\text { with coconut } \\
\text { oil and } \\
\text { applied on } \\
\text { breast. }\end{array}$ \\
\hline 40 & $\begin{array}{l}\text { Withania } \\
\text { somnifera( L.) } \\
\text { Dunal }\end{array}$ & Solanaceae & $\begin{array}{l}\text { Ashwagan } \\
\text { dha }\end{array}$ & Roots & Infertility in men & $\begin{array}{l}\text { Powdered root } \\
\text { with cow's } \\
\text { milk taken } \\
\text { orally for one } \\
\text { week. }\end{array}$ \\
\hline
\end{tabular}

\section{Conclusion}

The above list includes only those plant species which are of common occurrence and abundantly available in rural, semi-urban or even in urban areas. In our discussions with the vaidya or kaviraj, the latter emphasized the need for proper preparation and administration of these ayurvedic medicines. The specific part of the plant-roots, leaves, fruits or seeds, used in the preparation of drugs has to be taken care off. The various preparations were decoction, concoction, juice or dried and powdered plant parts. The powdered forms are usually recommended to be taken with madhu (honey). Honey besides making the preparations palatable also has its own therapeutic value. The practitioners of traditional medicine believe that honey enhances the qualitative properties of the preparations.

With rapid changes in the environment and life conditions it is common that in most of such investigations informants believe that more medicinal plants were in use in the past than now [6]. During the course of our investigations, it was observed that the knowledge regarding medicinal plants was gradually eroding among the rural populace.This was because of the gradual demise of the old generation of practitioners of traditional medicine called vaidya or kaviraj and unwillingness of the village youth to learn and carry forward this age old wisdom.

Yet another cause of concern is the fast declining species of medicinal plants both in terms of their numbers and diversity. The major factors responsible for this are deforestation, biological invasion, agricultural expansion, overgrazing, drought, flood and fire. Many species are extinct now or are on the verge of extinction before their medicinal values could be known [7].Thus there is an urgent need to conserve these plant species for sustainable use in future.

\section{Acknowledgement}

The authors want to express their sincere gratitude to the rural populace of Varanasi region . Besides, we are also grateful to the local vaidya (practitioners of traditional medicine) for sharing their valuable knowledge with us.

\section{Books :}

\section{References}

[1] Ben-Erik van Wyk and Michael Wink, Medicinal Plants of the World : an illustrated guide to important medicinal plants and their uses (Singapore:Times Edition,c2004).

[2] D.A.Possey and G.Dutfield, Beyond Intellectual Property : Towards Traditional Resource Rights for indigenous People and Local communities (Canada : International Development Research Centre,1996).

[3] E.M.Williamson, Major Herbs of Ayurveda (Churchill Livingstone,London,2002).

[4] J.F.Duthie, Flora of the upper Gangetic Plains (Government Printer,1903-1929).

[5] J.D.Hooker, The Flora of British India (L.Reeve and Company,London, Vol. I-VII,1872-1897).

Journal Papers :

[6] M.Giday, Z.Afsfaw, T.Elmqvist and Z.Woldu, An Ethnobotanical study of medicinal plants used by the Zay people in Ethiopia, Journal of ethnopharmacology 85, 2003, 43-52.

[7] S.Biswas, Rare and threatened taxa in the forest flora of Tehri Garhwal Himalaya and strategies for their conservation, Indian Journal of forestry II (3),1998, 233-237. 